\title{
MIXED DARK MATTER FROM AXINO DISTRIBUTION
}

\author{
S.A. Bonometto ${ }^{1,2}$, F. Gabbiani ${ }^{3}$ and A. Masiero ${ }^{4}$
}

${ }^{1}$ Dipartimento di Fisica dell'Universitá di Milano, Via Celoria 16, I-20133 Milano, Italy

I.N.F.N. - Sezione di Milano

Dept. of Physics and Astronomy, University of Massachusetts at Amherst, Amherst, MA 01003, USA

I.N.F.N. - Sezione di Padova, Via Marzolo 8, I-35131 Padova, Italy

\section{Abstract}

We study the possibility of mixed dark matter obtained through the phase space distribution of a single particle. An example is offered in the context of SUSY models with a Peccei-Quinn symmetry. Axinos in the $100 \mathrm{keV}$ range can naturally have both thermal and non-thermal components. The latter one arises from the lightest neutralino decays and derelativizes at $z \sim 10^{4}$.

PACS number(s): 95.35.+d, 98.80.-k 


\section{Introduction}

Large scale structure data favour hybrid cosmological models. That dark matter (DM) consists of different components is an idea which dates back to the middle eighties, when the linear stages of hybrid models were fully evaluated ${ }^{(1)}$. The failure of canonical cold dark matter (CDM) models recently renewed the interest on such models. A recent evaluation of their non-linear stages ${ }^{(2)}$ shows that it eases the solution of the problems CDM could not solve. Recent $\operatorname{work}^{(3)}$ is based on a mixture of massive neutrinos with $m_{\nu} \simeq 7$ $\mathrm{eV}$ (hot dark matter: HDM) and other non-baryonic particles yielding CDM. The HDM density parameter $\Omega_{\nu}=0.3\left(m_{\nu} / 28 \mathrm{~h}^{2} \mathrm{eV}\right.$ ) (h: Hubble parameter in units of $100 \mathrm{~km} \mathrm{~s}^{-1} \mathrm{Mpc}^{-1}$, in this note we take $h^{2}=0.5$ ). Baryonic matter, expected to have a marginal role in the origin of inhomogeneities, has a density parameter $\Omega_{\mathrm{B}} \lesssim 0.05$, because of primeval nucleosinthesis constraints. The cold component is then assumed to have a density parameter $\Omega_{\mathrm{CDM}} \simeq 0.6$, in order that $\Omega=1$. Massive neutrinos derelativize at a redshift $z_{\mathrm{der}} \simeq 10^{4}\left(m_{\nu} / 7 \mathrm{eV}\right)$

With respect to canonical CDM, the above CHDM hybrid model has only one extra parameter $\left(m_{\nu}\right)$. Nevertheless it is hard to escape the feeling that having $\Omega_{\mathrm{HDM}} / \Omega_{\mathrm{CDM}}$ of $\mathrm{O}(1)$ is a fortuitous coincidence. In this note we propose a hybrid model where a single particle naturally accomplishes the twofold role of CDM and HDM, thanks to its expected phase space distribution. The value of the $\Omega_{\mathrm{HDM}} / \Omega_{\mathrm{CDM}}$ ratio is linked to precise microphysical parameters. For a significant sector of the parameter space, we expect our model to reproduce (or to improve) CHDM outputs. It is clear that the quality of a model cannot be strictly linked to the request of minimizing the total parameter amount. Our choice of microphysical parameters is fairly natural and testable at present or forseeable experimental facilities.

Our framework is based on the supersymmetric (SUSY) extension of the standard model where a PecceiQuinn (PQ) symmetry ${ }^{(4)}$ is implemented to solve the strong CP problem. More specifically, we consider a SUSY version of the invisible DFS axion ${ }^{(5)}$. Accordingly, beside axions, the model predicts the existence of its fermionic partner called axino $(\tilde{a})$.

In order to obtain MDM, such framework is to be restricted as follows: (i) The lightest neutralino $(\chi)$ is an (almost) pure gaugino. (ii) The SUSY soft breaking scale, related to the sfermion $(\tilde{f})$ masses is of $\mathrm{O}(\mathrm{TeV})$. (iii) The PQ scale $\left(V_{\mathrm{PQ}}\right)$ is in the lower side of the interval allowed by astrophysical constraints ${ }^{(6)}$, ranging about $10^{10} \mathrm{GeV}$.

As the axino mass $m_{\tilde{a}} \sim m_{\tilde{f}}^{2} / V_{\mathrm{PQ}}$, taking $V_{\mathrm{PQ}} \sim 10^{10} \mathrm{GeV}$ and $m_{\tilde{f}} \sim \mathrm{TeV}$, leads to $m_{\tilde{a}}$ of $\mathrm{O}(100 \mathrm{keV})$. In turn this would be coherent only with a present CBR temperature $T_{o} \ll 2.7 \mathrm{~K}$ (or, if we require $T_{o}=2.7 \mathrm{~K}$, with $\Omega \gg 1$, well above observational limits) unless a substantial entropy release took place between axino decoupling and today, e.g. at the electroweak phase transition ${ }^{(7)}$. Such release rises the CBR temperature at the observed level.

In two previous works it was already suggested that DM consists of $\tilde{a}$ 's. In one of them ${ }^{(8)}$ it was assumed that DM $\tilde{a}$ 's originated because of $\chi$ decay. In the other one ${ }^{(9)}$ it was shown that this could hardly account for $\Omega=1$, while $\tilde{a}$ 's, formerly in thermal equilibrium with the other components of the Universe and decoupling at a temperature $T_{\tilde{a}, d g}<V_{\mathrm{PQ}}$, can easily account for $\Omega=1$. In this note we take into account both thermal and non-thermal axino components. The former component will be an effective CDM, as only fluctuations involving masses $\mathrm{M} \lesssim 0.1 \mathrm{M}_{\odot}$ will be erased at its derelativization. The latter component, instead, can easily behave as HDM, derelativizing at a redshift $z \sim 10^{4}$. Accordingly, fluctuations in such component will be erased up to a mass $\mathrm{M}_{\mathrm{D}} \sim 10^{15} \mathrm{M}_{\odot}$. The exact values of the ratio $\Omega_{\mathrm{HDM}} / \Omega_{\mathrm{CDM}}$ and of $z_{\text {der }}$ depend on $m_{\chi}$ (neutralino mass), $m_{t}$ (top quark mass), and $m_{\tilde{f}}$.

\section{Non-thermal Axino Production}

Axinos are the natural lightest SUSY fermions; therefore $\chi$ 's can decay, according to the reaction $\chi \rightarrow \tilde{a}+\gamma$ (we assume R-parity to be conserved). The temperature of the Universe when this occurs is given by:

$$
T_{d y}^{4}=45 m_{p l}^{2} \Gamma_{d y}^{2} / 4 \pi^{3} g_{\chi, d y}
$$


Here $m_{p l}=G^{-1 / 2}=1.2 \times 10^{19} \mathrm{GeV}, g_{\chi, d y}=\mathcal{N}_{b}+(7 / 8) \mathcal{N}_{f}\left[\mathcal{N}_{b(f)}\right.$ is the number of independent spin states of bosons (fermions) of particles with $m<T_{d y}$, still coupled at $\left.T_{d y}\right]$, and

$$
\Gamma_{d y}=\left(\alpha_{\mathrm{em}}^{2} / 18 \pi^{3}\right)\left(m_{\chi}^{3} / V_{\mathrm{PQ}}^{2}\right)|f(y)|^{2}
$$

is the decay rate ${ }^{(10)}$, which is equated to the cosmic expansion rate. In eq. (2) $f(y)=y(1-y+\ln y) /(1-y)^{2}$

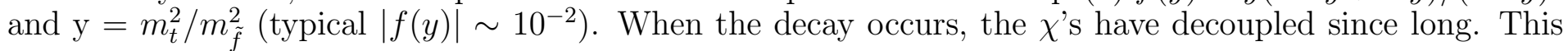
occurred at a temperature $T_{\chi, d g}$ when $x=T_{\chi, d g} / m_{\chi}$ was already below unity: $x$ is obtained equating the cosmic expansion rate with the rate of the reaction $\chi \chi \rightarrow f \bar{f}$. Assuming that $\chi$ is a pure gaugino is crucial to restrict the dynamics of the reaction to pure sfermion exchange (we consider Higgs exchange contribution to be negligible). According to ref. (11) we obtain

$$
e^{1 / x} x^{-3 / 2}=\left(8 \alpha_{\mathrm{em}}^{2} / \pi^{2}\right)\left(45 / 2 g_{\chi, d g}\right)^{1 / 2} N m_{\chi}^{3} m_{p l} / m_{\tilde{f}}^{4},
$$

with $N=116 / 27=\sum_{f} q_{f}^{4} n_{c, f}\left(q_{f}\right.$ : fermion charge; $n_{c}$ : color number), while $g_{\chi, d g}$ is the analogue of $g_{\chi, d y}$, but at $T_{d g}$ instead of $T_{d y}$. The number density of residual $\chi$ 's is then

$$
n_{\chi, d g}=0.70 n_{\text {rel }}\left(T_{\chi, d g}\right) x^{-3 / 2} e^{-1 / x},
$$

where $n_{\text {rel }}(T)=\left(\zeta(3) / \pi^{2}\right) g_{n} T^{3}$, while $g_{n}=\mathcal{N}_{b}+(3 / 4) \mathcal{N}_{f}$ (therefore, in our case, $\left.g_{n}=1.5\right)$. The number of $\tilde{a}$, arising from $\chi$ decay, at any $T \ll T_{\chi, d y}$, is $n_{\tilde{a}}=n_{\chi, d g}\left[a_{\chi, d g} / a(T)\right]^{3}$, a being the scale factor, while their linear momentum, in average, is $\left(m_{\chi} / 2\right)\left[a_{\chi, d y} / a(T)\right]$. The detailed momentum distribution is quite far from thermal. It can be easily obtained taking into account that each $\tilde{a}$, when it arises (from non-relativistic $\chi$ 's), has momentum $p=m_{\chi} / 2$. Let $t^{*}$ be the time when a given $\tilde{a}$ is produced, the comoving number of $\chi$ 's then reads

$$
n_{\chi}=n_{\chi, d g} \exp \left(-2 \Gamma_{d y} t^{*}\right) .
$$

At any time $t \ll \Gamma_{d y}^{-1}$, the momentum distribution of $\tilde{a}$ 's is obtainable from the relation

$$
\frac{d n_{\tilde{a}}}{d p}=-2 \frac{t^{*}}{z^{*}} \frac{d n_{\tilde{a}}}{d t^{*}} \frac{d z^{*}}{d p}
$$

(redshift and scale factor are related according to $1+z=a_{o} / a$ ). Here $p=m_{\chi} z / 2 z^{*}$ is the momentum of $\tilde{a}$ 's at the time $t ; z$ and $z^{*}$ are the redshifts at the times $t$ and $t^{*}$ respectively. Owing to eqs. (5) and (6), the distribution of $\tilde{a}$ 's on momentum, at the redshift $z$, reads

$$
\Phi_{z}(p)=\frac{1}{n_{\chi, d g}} \frac{d n_{\tilde{a}}}{d p}=2 \frac{q}{p} e^{-q}
$$

with $q=\left[\left(z_{\chi, d g} / z\right)\left(2 p / m_{\chi}\right)\right]^{2}$. For $p=m_{\tilde{a}}$ axinos derelativize. In average, this takes place at a redshift given by

$$
z_{\mathrm{der}} / 10^{4} \simeq 0.2|f|\left(m_{\tilde{a}} / k e V\right)\left(m_{\chi} / 10 \mathrm{GeV}\right)^{1 / 2}\left(V_{\mathrm{PQ}} / 10^{10} \mathrm{GeV}\right)^{-1}
$$

Eq. (8) immediately shows that, taking $m_{\chi}$ and $m_{t}$ (from which the value of $f$ follows) in the experimentally admitted range, and choosing low $V_{P Q}$ and high $m_{\tilde{f}}$ (from which the value of $m_{\tilde{a}}$ follows), $z_{\text {der }} / 10^{4} \simeq 1$ is a generic consequence. Quite independently of their mass and because of their non-thermal distribution, axinos derelativize at the same epoch as massive neutrinos with mass $\sim 7 \mathrm{eV}$, and a previously thermal distribution.

Together with the right derelativization, axinos also have a fair number density. Let us stress that $n_{\chi}$ and, therefore, $n_{\tilde{a}}$ have a strong dependence on $m_{\tilde{f}}$. This can be seen through eqs. (3) and (4). If powers of $x$ are neglected with respect to exponentials, it turns out that $n_{\tilde{a}} \propto m_{\tilde{f}}^{4}$. With the large values of $m_{\tilde{f}}$ 
considered here, $\chi$ 's in the allowed mass range would close the Universe, unless they decay into $\tilde{a}$ (or other particles with masses $\left.\lesssim 10^{3} \mathrm{keV}\right)$.

Let us also notice that, once we require that $m_{\chi} / m_{\tilde{a}} \gtrsim 10^{5}$, having $z_{\text {der }} \sim 10^{4}$ leads to $z_{\chi, d y} \gtrsim 10^{9}$. However, the value of $z_{\chi, d y}$ is soon obtainable from eq. (2). The point is that, within the above range of $V_{P Q}$ and $m_{\tilde{f}}$, the right value of $\Gamma_{d y}$ follows from taking the top mass in the $150 \mathrm{GeV}$ range, according to experimental constraints.

Decay photons, emitted at $z \sim 10^{9}(T \sim 230 \mathrm{keV})$ are thermalised and cause no observable distortion of CBR spectrum. No appreciable amount of nuclides, produced in big-bang nucleosynthesis, is likely to be destroyed by decay photons. We shall devote the rest of this section to show this point. The essential argument is that, at least down to $T \sim 20 \mathrm{keV}$, nuclides are screened against $\chi$-decay photon disruption either by CBR photons or by the residual thermal electrons. Then, at $T<20 \mathrm{keV}$, the residual number of $\chi$ is however too small to cause any appreciable consequence.

At any given temperature $T<230 \mathrm{keV}$, the residual number density of $\chi$ reads

$$
n_{\chi}(T) \sim 10^{-5} n_{\gamma} e^{-(230 \mathrm{keV} / T)^{2}} .
$$

Here $n_{\gamma}$ is the thermal photon number density at the same $T$. (If $m_{\chi}$ exceeds $30 \mathrm{GeV}, n_{\chi}$ would be even smaller.) Photons arising from $\chi$ decay are well above threshold for the production of $e^{+}, e^{-}$pairs in the collision with CBR. At $T \sim 100 \mathrm{keV}$ the cross-section for this process is $\sim 10^{-3} \sigma_{T}\left(\sigma_{T}\right.$ : Thomson cross section), while the cross-section for the reaction $\gamma+{ }^{2} \mathrm{H} \rightarrow p+n$ is $\sim 10^{-6} \sigma_{T} \cdot{ }^{2} \mathrm{H}$ disruption by high energy $\gamma$ 's is therefore a negligible process.

However, this is not enough to show that nuclide disruption is unlikely. In fact, in the $e^{+}, e^{-}$pair production most energy goes to a single electron. Such electron, via inverse-compton in the Klein-Nishina regime, is going to yield almost all its energy to another photon, etc. This creates a cascade process ${ }^{(12)}$ and the low energy by-products, both electrons and $\gamma^{\prime}$ s, are potentially dangerous to ${ }^{2} \mathrm{H}$, which is no longer shielded by CBR $\gamma^{\prime} s$, as low energy by-products are mostly below threshold for $e^{+}, e^{-}$pair production.

As a matter of fact, at $T<m_{e}$, the thermal electron abundance is depressed by a Boltzmann factor $\sim y^{3 / 2} e^{-y}$ with $y=m_{e} / T$, but, at $T \sim 100 \mathrm{keV}$, a considerable amount of electrons are still present. Only when $T \sim 20 \mathrm{keV}(y \sim 25)$ does the electron number density become so low, to approach the baryon number density. Let us however recall that the cross section for nuclide disruption is $\sim 10^{-6} \sigma_{T}$. The number of cascade by-products per $\chi$-decay is smaller than $10^{6}$ and therefore residual electrons are a good shield to ${ }^{2} \mathrm{H}$ and other nuclides. At $T \sim 20 \mathrm{keV}$, the residual $n_{\chi} \sim 10^{-48} n_{\gamma}$. This compares with the small abundances of light nuclides produced in big-bang nucleosynthesis. E.g., the predicted ${ }^{7} \mathrm{Li}$ abundance corresponds to a number density $n_{\left[{ }^{7} L i\right]} \sim 10^{-18} n_{\gamma}$ and cannot be appreciably modified by any photon cascade caused by $\chi$ decay.

\section{Thermal Axino Production}

Aside of non-thermal $\tilde{a}$ 's, thermal $\tilde{a}$ 's, decoupling when the cosmic temperature $T_{\tilde{a}, d g}$ was slightly below $V_{\mathrm{PQ}}$, are expected to exist ${ }^{(9)}$. Their present number density can be obtained from

$$
a_{\tilde{a}-d g}^{3} T_{\tilde{a}-d g}^{3}=a_{\tilde{a}-d g}^{3}\left[\pi^{2} / \zeta(3) g_{n, \tilde{a}}\right] n_{\tilde{a}, \tilde{a}-d g}=a_{o}^{3}\left[\pi^{2} / \zeta(3) g_{n, \tilde{a}}\right] n_{\tilde{a}, o} .
$$

$\left(g_{n, \tilde{a}}=3 / 2\right)$. After $\tilde{a}$ decoupling, the Universe underwent (at least) the electroweak phase transition. If we consider the entropy increase ${ }^{(7)}$ at such transition(s), current mass limits for particles decoupling earlier are modified. We shall take that into account by means of a suitable $\mu$ factor in the following relation:

$$
\mu\left(g_{\tilde{a}-d g}-g_{\tilde{a}}\right) a_{\tilde{a}-d g}^{3} T_{\tilde{a}-d g}^{3}=g_{o} a_{o}^{3} T_{\gamma, o}^{3}=\left(g_{o} / 2\right)\left[\pi^{2} / \zeta(3)\right] a_{o}^{3} n_{\gamma, o} .
$$

Here $g_{o}=g_{\gamma}\left(g_{\nu}+g_{\gamma}+g_{e}\right) /\left(g_{\gamma}+g_{e}\right)=43 / 11\left(n_{\gamma, o}\right.$ is the present photon number density). Comparing eqs. (10) and (11), after simple calculations, yields the present density parameter $\Omega_{\mathrm{CDM}}$ of thermal $\tilde{a}$. This reads

$$
\Omega_{\mathrm{CDM}}=0.51 \frac{m_{\tilde{a}}}{\operatorname{keV}} \frac{1}{\mu h^{2}} \frac{220}{\left(g_{\tilde{a}-d g}-g_{\tilde{a}}\right)} .
$$


The spin degrees of freedom at $T_{\tilde{a}, d g}$ yield $g_{\tilde{a}, d g} \simeq 220$. Both non-thermal and thermal $\tilde{a}$ 's are fully nonrelativistic today. Accordingly their present densities are $\rho_{o, \tilde{a}-n . t h}=m_{\tilde{a}} n_{\chi, \chi-d g}\left(a_{\chi-d g} / a_{o}\right)^{3}$ and $\rho_{o, \tilde{a}-t h}=$ $m_{\tilde{a}} n_{\tilde{a}, \tilde{a}-d g}\left(a_{\tilde{a}-d g} / a_{o}\right)^{3}$. Then, owing to eqs. (4) and (12), we have that

$$
\frac{\Omega_{\mathrm{HDM}}}{\Omega_{\mathrm{CDM}}} \simeq \frac{0.70 g_{\chi}\left(g_{\tilde{a}-d g}-g_{\tilde{a}}\right) \mu}{g_{\tilde{a}} g_{\chi-d g}} x^{-3 / 2} e^{-1 / x} .
$$

In the above relations the microphysical variables are $m_{\tilde{a}}, m_{\chi}, m_{\tilde{f}}, m_{t}, V_{\mathrm{PQ}}$. Other physical variables are $\mu$, $\Omega_{\mathrm{CDM}}, \Omega_{\mathrm{HDM}}$ and $z_{\mathrm{der}}$. Besides of eqs. (8), (11), (12), the constraint $m_{\tilde{a}} V_{\mathrm{PQ}} \simeq m_{\tilde{f}}^{2}$ also holds.

Before concluding this section we wish to outline that the factor $\mu$ introduced here is also related to the baryon-to-photon ratio after the electroweak transition (where baryon number could be generated, if an out-of-equilibrium phase lasts long enough). In principle this might allow to relate the final value of the present baryon density parameter $\Omega_{B}$ to the final ratio $\frac{\Omega_{\mathrm{HDM}}}{\Omega_{\mathrm{CDM}}}$.

\section{Conclusions}

Within the frame of a SUSY implementation of the invisible DFS axion approach, we can restrict the parameter space either considering the expected ranges for $m_{\chi}, m_{\tilde{f}}, m_{t}$ and $V_{\mathrm{PQ}}$, or by requiring that $z_{\text {der }} \sim 10^{4}, \Omega_{\mathrm{CDM}} \sim 0.6-0.8, \Omega_{\mathrm{CDM}} / \Omega_{\mathrm{HDM}} \sim 3-4$. The point we wish to make is that there is a significant overlap between the parts of the parameter space which are allowed by either set of constraints. We illustrate this fact by means of figs. 1-2 where microphysical parameters are constrained to provide suitable values of $z_{\mathrm{der}} . \Omega_{\mathrm{CDM}}=0.8$ and $\Omega_{\mathrm{HDM}}=0.2$ are taken, but results do not depend critically on this assumption.

Fig. 1 shows for which values of $m_{\tilde{f}}$ and $m_{\chi}$ we obtain $z_{\text {der }} \simeq 10^{4}$. Different curves refer to the top masses ranging from $120 \mathrm{GeV}$ to $180 \mathrm{GeV}$. Fig. 2 shows the dependence of the $V_{\mathrm{PQ}}$ scale on $m_{\chi}$, for $m_{t}=$ $140 \mathrm{GeV}$ and requiring $z_{\mathrm{der}} \simeq 10^{4}$. For $m_{\chi} \simeq 30 \mathrm{GeV}, V_{\mathrm{PQ}} \sim 1.7 \times 10^{10} \mathrm{GeV}$. The corresponding value of $m_{\tilde{a}}$ is $\sim 180 \mathrm{keV}$, while $\mu \sim 220$. Fig. 3 is a description of the evolution of densities for different components in the Universe, starting from $T \sim V_{\mathrm{PQ}}$ down to today's temperature. This plot is meant to show how complicate it can appear, a priori, to build up a component arising from heavier particle decay, with both the right derelativization redshift $\left(z_{d e r}\right)$ and final density parameter $\left(\Omega_{\mathrm{HDM}}\right)$. The main issue of this paper is that such aims are achieved fairly naturally in a SUSY implementation of the DFS invisible axion approach, provided large SUSY scale and small PQ scale are taken.

The detailed evolution of density fluctuations over different scales, through $\tilde{a}$ derelativization, equivalence, hydrogen recombination should be computed in order to evaluate CBR fluctuations, large scale structure and velocity fields, galaxy mass function. A study of the linear stages of this model will be presented elsewhere. It is however possible to estimate the minimal mass of fluctuations able to survive derelativization both for the cold and the hot parts of $\tilde{a}$ distribution ( $M_{D, c}$ and $M_{D, h}$, respectively), just by evaluating the mass scales entering the horizon at the time $t$ of derelativization.

For the cold component, $M_{D, c} \simeq\left(2 \pi^{3} / 45\right) g_{\tilde{a}} T_{\tilde{a}}^{4} t^{3}$, while $t=\left(45 / 8 \pi^{3} g_{o}\right)^{1 / 2} m_{p l} T^{-2}$; the relation between $\tilde{a}$ temperature and photon temperature is $T_{\tilde{a}}=\left(g_{o} / g_{\tilde{a}-d g} \mu\right)^{1 / 3} T$. Accordingly, requiring $T_{\tilde{a}}=m_{\tilde{a}}$, we have that

$$
M_{D, c} \simeq\left(\frac{45}{128 \pi^{3}}\right)^{1 / 2} \frac{g_{\tilde{a}} g_{o}^{1 / 2}}{g_{\tilde{a}-d g}^{2} \mu^{2}} \frac{m_{p l}^{3}}{m_{\tilde{a}}^{2}}
$$

is $\sim 0.1 M_{\odot}$. Such small value of $M_{D, c}$ is due to the actual $\tilde{a}$ temperature.

On the contrary $M_{D, h} \simeq\left(\pi^{2} / 40\right) t_{o} z_{\text {der }}^{-3 / 2} m_{p l}^{2} \simeq 3.6 \times 10^{15}\left(10^{4} / z_{\text {der }}\right)^{1.5} M_{\odot}$. Non thermal $\tilde{a}$ distribution has a wide energy spread. Derelativization can be therefore expected to occur more gradually than for standard $7 \mathrm{eV}$ neutrinos.

In the context of a phenomenologically viable SUSY model it is therefore possible to obtain a phase space distribution of a single particle which provides a valid hybrid cosmological model. 


\section{Acknowledgements}

Thanks are due to David Lyth, Michael Dine and Joel Primack for discussions. F.G. thanks the Departments of Physics of Milano and Padova Universities for their kind hospitality.

\section{References}

1) R. Valdarnini and S.A. Bonometto, Astr. \& Astroph., 146, 235 (1985); S.A. Bonometto and R. Valdarnini, Astroph. J. 299, L71 (1985); see also Q. Shafi and F.W. Stecker, Phys. Rev. Lett. 53, 1292 (1984) and S. Achilli, F. Occhionero and R. Scaramella, Astroph. J. 299, 577 (1985).

2) A. Klypin, J. Holtzman, J. Primack and E. Regös, Astroph. J. 416, 1 (1993).

3) J.A. Holtzman, Astroph. J. Suppl. 71, 1 (1989); A.N. Taylor and M. Rowan-Robinson, Nature 359, 396 (1992); J.A. Holtzman and J. Primack; Astrophys. J. 396, 113 (1992); a recent note by D.Yu. Pogosyan and A.A. Starobinski, CUA preprint (1993), shows that an hybrid model with $\Omega_{\mathrm{HDM}} \sim 0.2$, while keeping $z_{\text {der }} \sim 10^{4}$, would provide an even better fit of data.

4) R. Peccei and H. Quinn, Phys. Rev. Lett. 38, 1440 (1977); Phys. Rev. D 16, 1791 (1977).

5) M. Dine, W. Fischler and M. Srednicki, Phys. Lett. 104 B, 99 (1981).

6) D.A. Dicus, E.W. Kolb, V.L. Teplitz and R.V. Wagoner, Phys. Rev. D 22, 839 (1980); M. Fukugita, S. Watamura and M. Yoshimura, Phys. Rev. D 26, 1840 (1981).

7) This occurred if such transition took place after a suitable supercooling and as a deflagration in a regime next to the Jouguet curve. See K. Enqvist, J. Ignatius, K. Kajantie and K. Rummukainen, Phys. Rev. D 45, 3415 (1992). The amount of entropy released depends on the length of expansion during supercooling. $T_{o}$ would rise to the observed value if the increase of the scale factor is $\sim 6$.

8) S.A. Bonometto, F. Gabbiani and A. Masiero, Phys. Lett. B 222, 433 (1989).

9) K. Rajagopal, M.S. Turner and F. Wilczek, Nucl. Phys. B 358, 447 (1991).

10) J.F. Nieves, Phys. Lett. B 174, 411 (1986). For a previous calculation in the limit of equal top and stop masses, see: J.E. Kim, A. Masiero and D.V. Nanopoulos, Phys. Lett. 139 B, 346 (1984).

11) J. Ellis et al., Nucl. Phys. B 238, 453 (1984).

(12) See, e.g., S.A. Bonometto, Lett. Nuovo Cimento 1, 677 (1971), and S.A. Bonometto, F. Lucchin and P. Marcolungo, Astr. \& Astrophys. 31, 41 (1974).

\section{Figure Captions}

Fig. 1 - Values of $m_{\tilde{f}}$ and $m_{\chi}$ leading to $z_{\mathrm{der}} \simeq 10^{4}$ for $\Omega_{\mathrm{CDM}}=4 \Omega_{\mathrm{HDM}}=0.8$. Different curves (bottom to top) refer to $m_{t}$ from $120 \mathrm{GeV}$ to $180 \mathrm{GeV}$.

Fig. $2-V_{\mathrm{PQ}}$ as a function of $m_{\chi}$ for $m_{t}=140 \mathrm{GeV}$ and $z_{\mathrm{der}} \simeq 10^{4}$.

Fig. 3 - Evolution of densities of radiation (solid line), CDM (dashed line) and HDM (dotted line) in logarithmic scales. The HDM line accounts for $\chi$ 's prior to their decay and then for non-thermal axinos. The large increase of $\rho T^{-4}$ at $T \sim 100 \mathrm{GeV}$ is related to entropy release at the electroweak phase transition. 


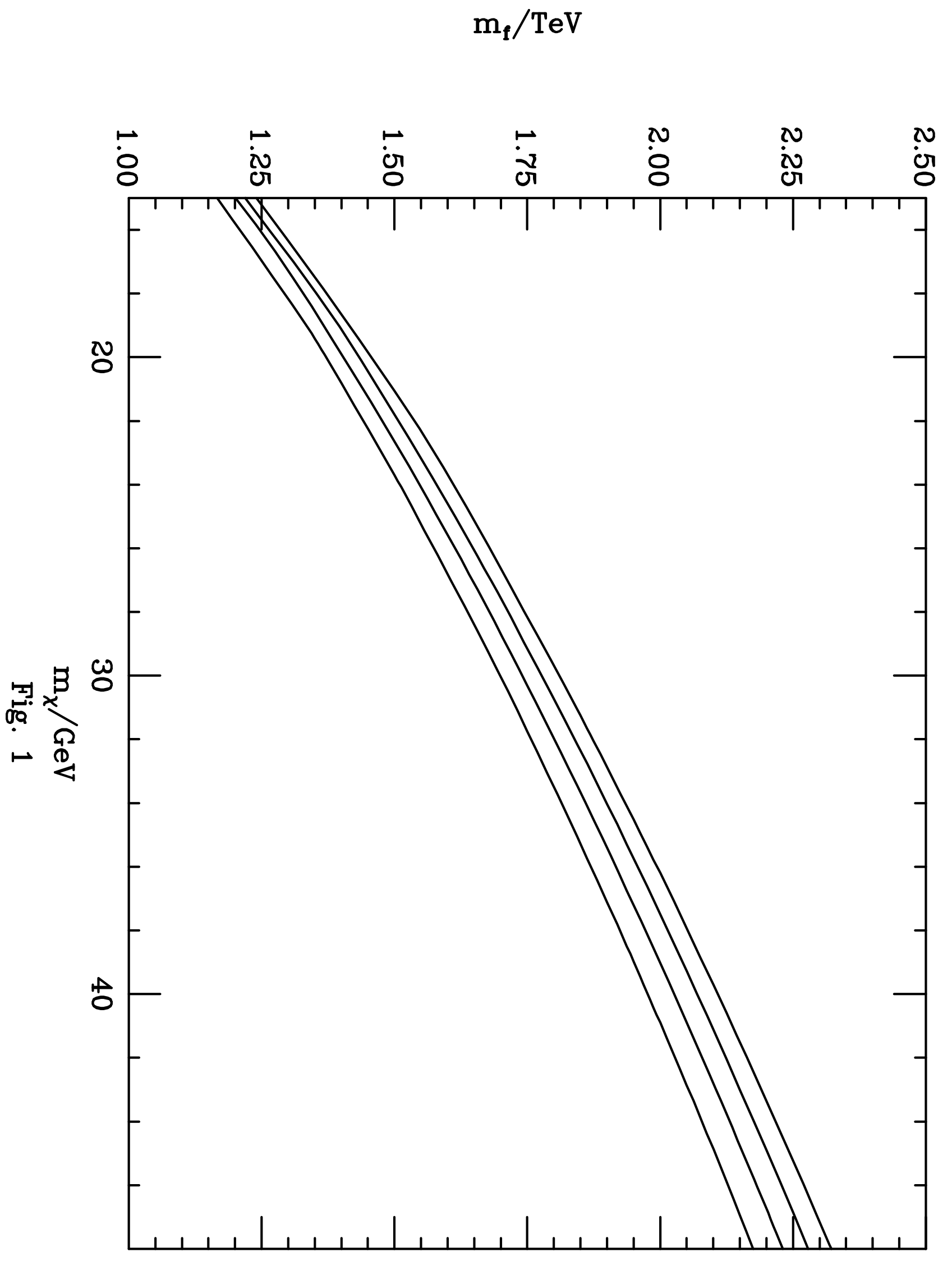




$$
\mathrm{V}_{\mathrm{PQ}} / 10^{10} \mathrm{GeV}
$$

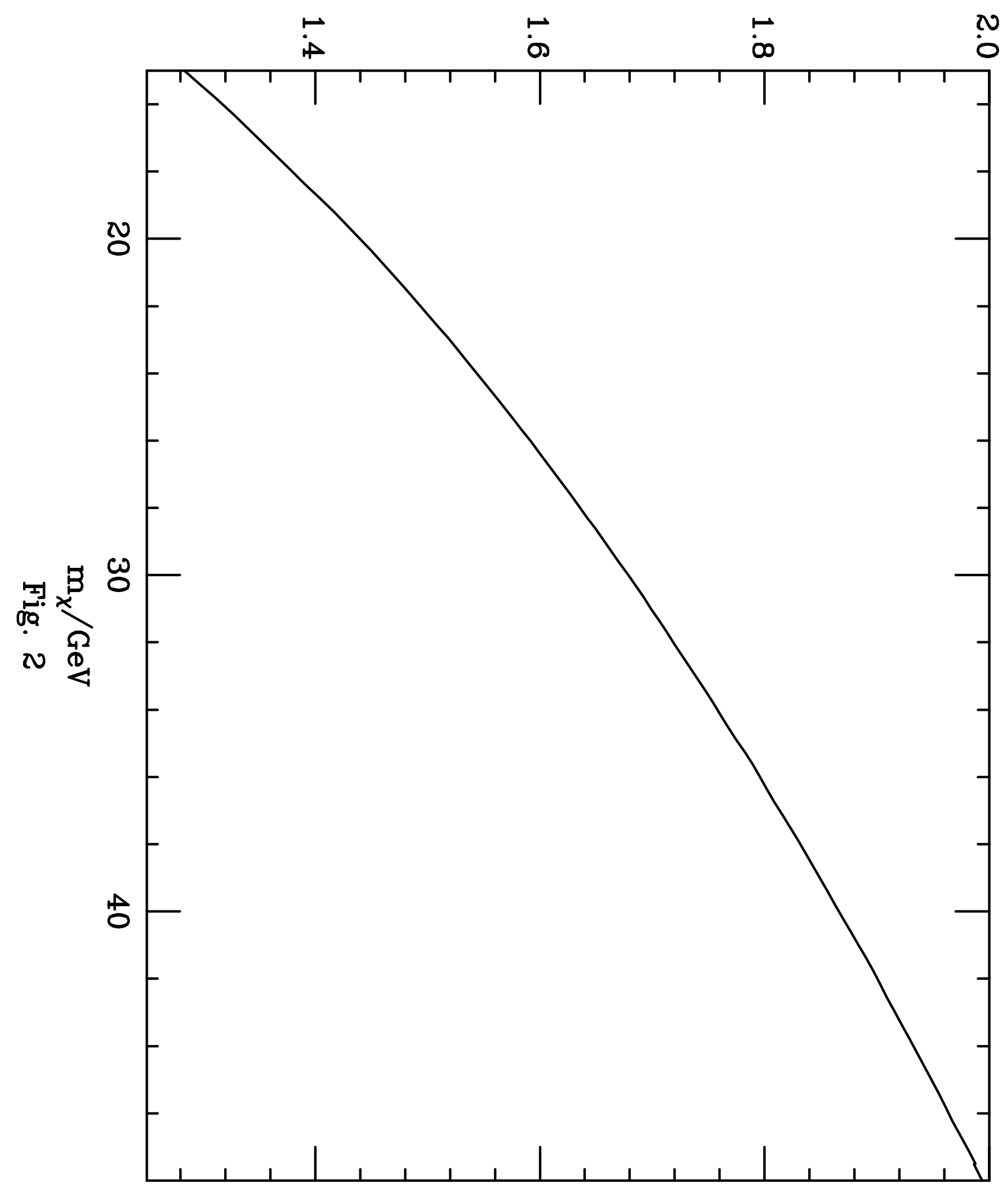


$\rho \mathrm{T}^{-4}$

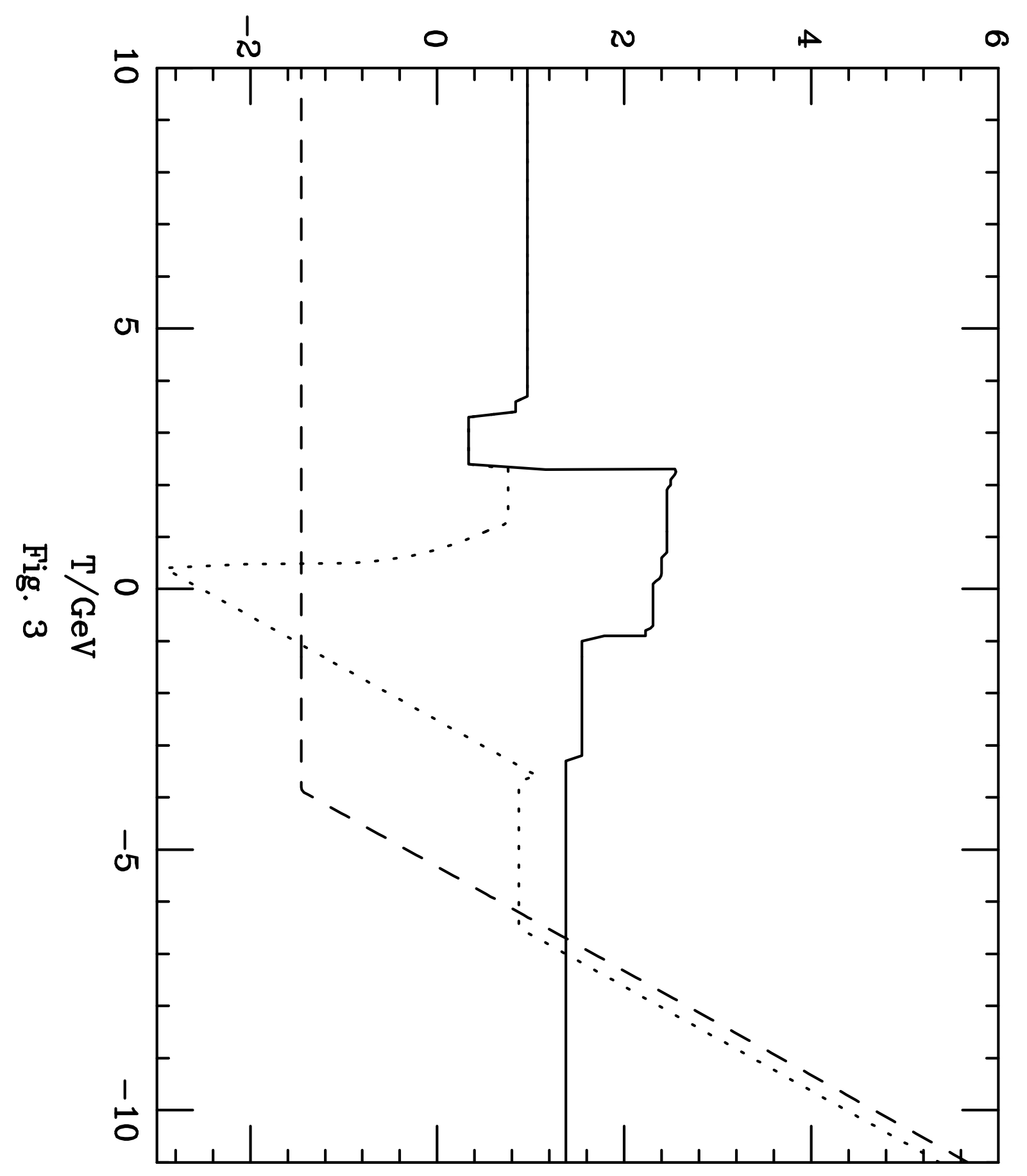

